\title{
Charge-monopole versus Gravitational Scattering at Planckian Energies
}

\author{
Saurya Das and Parthasarathi Majumdar \\ The Institute of Mathematical Sciences, CIT Campus, Madras 600113, India.
}

\begin{abstract}
The amplitude for the scattering of a point magnetic monopole and a point charge, at centre-of-mass energies much larger than the masses of the particles, and in the limit of low momentum transfer, is shown to be proportional to the (integer-valued) monopole strength, assuming the Dirac quantization condition for the monopole-charge system. It is demonstrated that, for small momentum transfer, charge-monopole electromagnetic effects remain comparable to those due to the gravitational interaction between the particles even at Planckian centre-of-mass energies.
\end{abstract}

14.80.Hv, 12.25.+e

Typeset using REVTEX 
The amplitude due to graviton exchange between two particles colliding at Planckian centre-of-mass $(\mathrm{cm})$ energies and in the limit of vanishingly small momentum transfer has been shown [1], [2] to be calculable semi-classically, corresponding to the eikonal approximation of quantum gravity. This simplification originates from the decoupling of all physical graviton modes in this kinematical regime, and the pure gauge states left behind have been shown to be describable by a topological sigma model in $1+1$ dimensions with diffeomorphism invariance [3] resembling a string theory. The semi-classical amplitude found by 't Hooft also showed a striking resemblance to the Veneziano amplitudes of string theory. One significant aspect was the existence of poles in this amplitude at $\mathrm{cm}$ energies $G s=-i N$ (where $N$ is a positive integer) [4. If the particles carry electric charge, the amplitude is still calculable semi-classically, corresponding to a shift $G s \rightarrow G s+\kappa \alpha$ with $G$ being Newton's constant, $s$ the squared cm energy, $\kappa$ is any integer (positive or negative) and $\alpha$ is the fine structure constant. Thus, at the Planck scale, electromagnetic effects due to these charges constitute a minor perturbation on the gravitational effects. At sub-Planckian cm energies that are still large compared to the particle masses, the quantum electrodynamic eikonal approximation [5] has been exactly reproduced from the semi-classical amplitude, and the corresponding topological sigma model has been identified [6].

In this paper, we study the scattering of a Dirac magnetic monopole with a pointlike electric charge in the same kinematical limit. At sub-Planckian energies (i.e., in the absence of graviton exchange), the amplitude turns out, remarkably, to be proportional to the integer $n$ occuring in the Dirac quantization condition, modulo standard kinematical factors. Next we turn on gravity, and show that even when the cm energy becomes on the order of the Planck scale, the monopole-charge interaction contribution to the cross section remains almost as large as the gravitational contribution, the former being characterised by the monopole strength $n$ as compared to the energy dependent gravitational coupling strength Gs occuring in the latter. Another remarkable feature of the amplitude in the presence of magnetic charge is the existence of poles at $G s=-i\left(N+\frac{n}{2}\right)$, in contrast to poles at $G s=$ $-i N$ for two particle scattering, and akin to poles in three particle scattering amplitudes 
found by 't Hooft [4. We shall comment on this result towards the end of the paper.

Consider first the scattering of an ultra-relativistic monopole from a slowly-moving charged particle within a kinematical region defined by a $\mathrm{cm}$ energy $s>>m_{1,2}^{2}\left(m_{1,2}\right.$ are the rest masses of the particles) and momentum transfer $t \rightarrow 0$, where, $s$ and $t$ are the usual Mandelstam variables. This kinematical region corresponds to a situation where the particles scatter almost solely in the forward direction, and for all practical purposes behave as though they are massless [1]. Further, the scattering can be described in terms of the response of the 'target' charged particle to the electromagnetic 'shock' wave-front carried by the fast-moving monopole. Treating the shock wave-front as classical, the calculation of the scattering amplitude reduces to determining the overlap of the wavefunctions of the point charge evaluated before and after encountering the monopole shock front. Basically, the fields in the shock front produce a phase factor in the charge wave-function, which can be exactly computed.

To determine the fields due to an ultra-relativistic monopole, we first boost the vector potential of a static monopole along the positive $z$-axis to some velocity $\beta$, and then evaluate the limit of this potential as $\beta \rightarrow 1$, as in ref.s [1] and [6]. The vector potential of a static monopole carrying magnetic charge $g$ can be given in spherical polar coordinates, following Wu and Yang [7], by

$$
\begin{aligned}
\vec{A}^{I}=\frac{g}{r \sin \theta}(1-\cos \theta) \hat{\phi}, & 0 \leq \theta<\pi \\
\vec{A}^{I I}=\frac{-g}{r \sin \theta}(1+\cos \theta) \hat{\phi}, & 0<\theta \leq \pi .
\end{aligned}
$$

Observe that we have made the gauge choice $A^{0}=0$, and have chosen an orientation of our coordinates such that only the $x$ and $y$ components survive. We consider first the potential (回). Giving it a Lorentz boost of magnitude $\beta$ along the positive z- axis yields

$$
{ }^{\beta} A_{i}^{I}=\frac{-g \epsilon_{i j} r_{\perp}^{j}}{r_{\perp}^{2}}\left[1-\frac{z-\beta t}{R_{\beta}}\right],
$$

where, $r_{\perp}^{2}=x^{2}+y^{2}, R_{\beta}=\left[(z-\beta t)^{2}+\left(1-\beta^{2}\right) r_{\perp}^{2}\right]^{\frac{1}{2}}$ and $i, j=1,2$. Using the limit [6]

$$
\lim _{\beta \rightarrow 1} \frac{1}{R_{\beta}}=\frac{1}{\left|x^{-}\right|}-\delta\left(x^{-}\right) \ln \mu^{2} r_{\perp}^{2}
$$


(where $\mu$ is an arbitrary dimensional parameter, and $x^{ \pm}$are the usual light cone coordinates), we get

$$
\vec{A}_{0}^{I} \equiv \lim _{\beta \rightarrow 1}{ }^{\beta} A_{i}^{I}=\frac{2 g}{r_{\perp}} \theta\left(x^{-}\right) \hat{\phi}
$$

which is the vector potential of an ultra-relativistic monopole. Note that, due to the presence of the $\theta$ function, $\vec{A}_{0}^{I}$ is singular only along that part of the $\mathrm{z}^{-}$axis which is behind the monopole. Similarly, boosting $\vec{A}_{0}^{I I}$ one obtains an expression (as $\beta \rightarrow 1$ ) which is proportional to $\theta\left(-x^{-}\right)$and is hence singular along the z-axis ahead of the monopole. In other words, these boosted potentials continue to satisfy the Wu-Yang criteria [7] for non-singular potentials due to a monopole.

The electric and magnetic fields can be calculated from the above to yield

$$
\begin{aligned}
& B^{i}=\frac{2 g r_{\perp}^{i}}{r_{\perp}^{2}} \delta\left(x^{-}\right), \quad B^{z}=0 \\
& E^{i}=\frac{2 g \epsilon_{i j} r_{\perp}^{j}}{r_{\perp}^{2}} \delta\left(x^{-}\right), \quad E^{z}=0 .
\end{aligned}
$$

This shows that $\vec{A}_{0}^{I}$ is a pure gauge everywhere except on the null plane. Note that the same fields can be obtained using duality symmetry, i.e. by the transformation $\vec{E} \rightarrow \vec{B}$ and $\vec{B} \rightarrow-\vec{E}$ on the fields already obtained by Jackiw et al [6] for the scattering of two point charges. Furthermore, It is possible to choose a gauge in which only the light cone components $A_{ \pm}$of the boosted vector potential survive, with the transverse components vanishing everywhere, yielding the same field strengths as in eq. (5). We shall discuss this case later.

Next we proceed to compute the scattering amplitude; to this end, we first rewrite $\vec{A}_{0}^{I}$ as a total derivative in the following form:

$$
\vec{A}_{0}^{I}=2 g \theta\left(x^{-}\right) \nabla \phi
$$

where, $\nabla$ is the gradient operator in the transverse (i.e., $x-y)$ plane. Before the arrival of the monopole with its shock front, the electric charge is described by a wave function which is a plane wave, 


$$
\psi_{<}=\psi_{0} \quad \text { for } x^{-}<0
$$

Immediately after the shock front passes by, the wave function is also a plane wave but with a gauge potential dependent phase factor, i.e.,

$$
\psi_{>}=\exp \left(i e \int d x^{\mu} A_{\mu}\right) \psi_{0}^{\prime}
$$

Upon using eq. (6) for the gauge potential in (8), we obtain

$$
\psi_{>}=\exp [i 2 \operatorname{eg} \phi] \psi_{0}^{\prime} \quad \text { for } x^{-}>0
$$

The plane wave solutions $\psi_{0}$ and $\psi_{0}^{\prime}$ are related through the continuity requirement

$$
\psi_{<}=\psi_{>} \quad \text { at } x^{-}=0
$$

Assume now that the monopole-charge system obeys the Dirac quantization condition

$$
e g=\frac{n}{2}
$$

so that,

$$
\psi_{>}=e^{i n \phi} \psi_{0}^{\prime}
$$

This sort of phase factor in the almost-forward scattering of a monopole and a charge was first found by Goldhaber [8]. Expanding $\psi$ in plane waves a lá 't Hooft [1], we get

$$
\psi_{>}=\int d k_{+} d^{2} k_{\perp} A\left(k_{+}, \vec{k}_{\perp}\right) \exp \left[i \vec{k}_{\perp} \cdot \vec{r}_{\perp}-i k_{+} x_{-}-i k_{-} x_{+}\right]
$$

with the on shell condition $k_{+}=\left(k_{\perp}^{2}+m^{2}\right) / k_{-}$. Multiplying both sides of (13) by a plane wave and integrating over $x_{-}$, we have

$$
A\left(k_{+}, k_{\perp}\right)=\frac{\delta\left(k_{+}-p_{+}\right)}{(2 \pi)^{2}} \int d^{2} r_{\perp} \exp \left(i n \phi+\vec{q} \cdot \vec{r}_{\perp}\right),
$$

where $\vec{q} \equiv \vec{p}_{\perp}-\vec{k}_{\perp}$ is the transverse momentum transfer, $k$ and $p$ being the final and initial momenta respectively. After integrating over the angular variable $\phi$, and appropriate scaling of the radial coordinate, the integral over the latter in (14) reduces to

$$
\frac{1}{q^{2}} \int_{0}^{\infty} d \rho \rho J_{n}(\rho)
$$


where $J_{n}(\rho)$ is the Bessel function of order $n$. The integration over the radial coordinate is standard [9], yielding

$$
\left(\frac{1}{-t}\right) \frac{2 \Gamma\left(1+\frac{n}{2}\right)}{\Gamma\left(\frac{n}{2}\right)}
$$

with $t \equiv-q^{2}$ being the momentum transfer. Putting everything together and incorporating canonical kinematical factors, the scattering amplitude is given by

$$
f(s, t)=\frac{k_{+}}{2 \pi k_{0}} \delta\left(k_{+}-p_{+}\right)\left(\frac{n}{-t}\right)
$$

Thus, as already mentioned, the scattering amplitude is proportional to the monopole strength $n$. Note that we would obtain the same result if we had used the second of the gauge potentials in (2) and performed the Lorentz boost etc. One reason for this is that, the potentials, boosted to $\beta \approx 1$, are both gauge equivalent to a gauge potential $A_{\mu}^{\prime}$ given by

$$
\vec{A}_{\perp}^{\prime}=0=A_{+}^{\prime} ; A_{-}^{\prime}=2 g \phi \delta\left(x^{-}\right) \text {everywhere }
$$

One can show that this gauge potential produces the same scattering amplitude as in (16). Thus, it appears that one can ignore the Dirac string singularity associated with the monopole gauge potential, in the kinematical limit under consideration. Observe that this is not true for boost velocities that are subluminal.

Two remarks are in order at this point. First of all, the scattering of an ultra-relativistic electric charge from a slow-moving Dirac monopole can be shown to yield an identical result as (16). The easiest way to see this is to use the dual formalism wherein one introduces a gauge potential $A_{\mu}^{M}$ such that the dual field strength $\tilde{F}_{\mu \nu} \equiv \partial_{[\mu} A_{\nu]}^{M}$. If this gauge potential is used to define electric and magnetic fields, then it follows that the standard field tensor must satisfy a Bianchi identity, which would then imply that the gauge potential due to a point charge must have a Dirac string singularity. Further, the monopole will behave identically to the point charge of the usual formalism, so that our method above is readily adapted to produce identical consequences. Secondly, one can treat the scattering of two Dirac monopoles in the same kinematical limit exactly as in [6], using this dual formalism. 
This would yield a result identical to the one for the electric charge case, with $e$ and $e^{\prime}$ being replaced by $g$ and $g^{\prime}$, the monopole charges.

Next, we consider the effect of turning on the gravitational interaction between the particles. As shown by 't Hooft [1], the gravitational shock wave due to the fast-moving particle also produces an extra phase factor in the wave function of the slow-moving target particle. The net effect of the two shock waves then is to produce a phase factor that is given by the superposition of the individual phase factors. It follows that the integral in eq. (14) must be replaced by

$$
\int d^{2} r_{\perp} \exp \left[i\left(n \phi-G s \ln \mu^{2} r_{\perp}^{2}+\vec{q} \cdot \vec{r}_{\perp}\right)\right]
$$

Once again, the integration over $\phi$ is readily done, giving the integral

$$
\frac{1}{q^{2}} \int_{0}^{\infty} d \rho \rho^{1-2 i G s} J_{n}(\rho)
$$

The radial integration is again quite standard, and yields [9] an amplitude

$$
f(s, t)=\frac{k_{+}}{4 \pi k_{0}} \delta\left(k_{+}-p_{+}\right)\left(\frac{n}{2}-i G s\right) \frac{\Gamma\left(\frac{n}{2}-i G s\right)}{\Gamma\left(\frac{n}{2}+i G s\right)}\left(\frac{4}{-t}\right)^{1-i G s} .
$$

With this, one can easily show that the cross section

$$
\frac{d^{2} \sigma}{d \vec{k}_{\perp}^{2}} \sim \frac{1}{t^{2}}\left(\frac{n^{2}}{4}+G^{2} s^{2}\right)
$$

Clearly, for cm energies of the order of the Planck mass, the contribution to this cross section due to the monopole-charge electromagnetic interaction is of the same order of magnitude as that due to the gravitational interaction between the particles. This is dramatically different from the case when the particles only have electric charge, where the electromagnetic effects constitute a small perturbation on the gravitational effects at Planck scale.

It is also remarkable that the amplitude given in eq. (16) exhibits poles at $G s=-$ $i\left(N+\frac{n}{2}\right)$ as opposed to the poles at $G s=-i N$ found in the absence of magnetic charge. Following the argument given in ref. [4], therefore, the spectrum of 'bound states' would appear to admit states of half-odd integral spin (for odd values of the monopole quantum $n$ ), 
in addition to those of integral spin. This appears to be similar to the spectrum discerned in three-particle scattering amplitudes [4]. While it is well known [10] that a monopolecharge system obeying Dirac quantization carries half-odd integral spin, the relation of this to the spectrum of states above is not quite transparent. In any event, the existence of such states in the spectrum would tend to imply that the underlying string structure (if any) corresponds to a supersymmetric rather than a bosonic string. Perhaps this would become clearer if we were to follow Verlinde and Verlinde [3] in an attempt to identify a topological sigma model for the pure gauge states of the system. We hope to report on this elsewhere. 


\section{REFERENCES}

[1] G. t'Hooft, Phys. Lett. 198B, 61 (1987).

[2] D. Amati, M. Ciafaloni and G. Veneziano, Phys. Lett. 197B,81 (1987).

[3] H. Verlinde and E. Verlinde, Nucl. Phys. B371, 246 (1992).

[4] G. 'T Hooft, Nucl. Phys. B304 867 (1988).

[5] M. Lévy and J. Sucher, Phys. Rev. 186, 1656 (1969).

[6] R. Jackiw, D. Kabat and M. Ortiz, Phys. Lett. B 277, 148 (1992).

[7] T. T. Wu and C. N. Yang, Phys. Rev. D14 437 (1976).

[8] A. S. Goldhaber, Phys. Rev. 140, B1407 (1965).

[9] Y.L. Luke, Integrals of Bessels Functions (McGraw-Hill Book Company, New York, 1962).

[10] M. N. Saha, Ind. J. Phys., 10, 145 (1936). 